\title{
Acute vision loss and choroidal filling delay in the absence of giant-cell arteritis
}

This article was published in the following Dove Press journal:

Clinical Ophthalmology

13 September 2016

Number of times this article has been viewed

\author{
Ling Bei ${ }^{1}$ \\ Iris Lee ${ }^{2}$ \\ Michael S Lee \\ Greg P Van Stavern' \\ Collin M McClelland ${ }^{3}$ \\ 'Department of Ophthalmology \\ and Visual Neurosciences, \\ Washington University School of \\ Medicine, St Louis, MO, ${ }^{2}$ Casey \\ Eye Institute, Oregon Health and \\ Science University, Portland, OR, \\ ${ }^{3}$ Department of Ophthalmology and \\ Visual Neurosciences, University of \\ Minnesota, Minneapolis, MN, USA
}

Correspondence: Collin M McClelland Department of Ophthalmology and Visual Neurosciences, University of Minnesota, 420 Delaware Street - SE/MMC 493, Minneapolis, MN 55455, USA Email cmc@umn.edu

\begin{abstract}
Giant-cell arteritis (GCA) is a visually devastating disease that often progresses to severe bilateral vision loss if untreated. Diagnosis of GCA is made challenging by the protean nature of the disease and the lack of a simple test that is both highly sensitive and specific. Choroidal filling delay on fluorescein angiography (FA) has been touted as a highly characteristic feature of GCA-related vision loss, although knowledge of both the sensitivity and specificity of this finding remains unproven. We report our experience of delayed choroidal filling on FA in a series of seven patients referred to an academic neuro-ophthalmology practice due to concern for GCA. Despite the FA findings, our examination, diagnostic testing, and long-term follow-up excluded the diagnosis of GCA in all cases, suggesting that choroidal perfusion abnormalities may occur in the absence of GCA. When evaluating a patient for acute vision loss, the astute clinician must remain cognizant of the limitations of FA in the diagnosis of GCA.
\end{abstract}

Keywords: giant-cell arteritis, fluorescein angiography, delayed choroidal filling

\section{Introduction}

Giant-cell arteritis (GCA), also termed temporal arteritis, is the most common primary form of vasculitis. ${ }^{1}$ Presenting predominately in Caucasian patients, GCA shows a predilection for women, with a female:male ratio of $2: 1 .^{2} \mathrm{GCA}$ occurs almost exclusively in patients older than 50 years, and its incidence increases with age: incidence in the sixth decade of life is 10.6 per 100,000 , rising to 44.6 per 100,000 after the age of 80 years. $^{2}$ Vision loss in GCA is common and often severe.

Anterior ischemic optic neuropathy is a common cause of acute optic neuropathy, and the majority of cases represent nonarteritic anterior ischemic optic neuropathy (NAION) unrelated to GCA. Arteritic anterior ION (AAION) is the cause of GCArelated vision loss in 78\%-99\% of cases. ${ }^{3,4}$ While GCA-related AAION from vasculitic occlusion of the posterior ciliary arteries (PCAs) represents a small proportion of these cases, it is considered an ophthalmic emergency and must be distinguished quickly from its more benign counterpart - NAION. If unilateral ION from GCA goes untreated, fellow-eye involvement is common. Furthermore, untreated GCA may lead to serious cardiovascular, neurological, and gastrointestinal comorbidities. ${ }^{5-7}$ Prompt diagnosis and treatment with corticosteroids are essential for halting progression of vision loss and avoiding systemic vasculitic sequelae.

Many factors contribute to the clinical challenge of differentiating between NAION and AAION, including occult cases of GCA lacking systemic symptoms, the limited specificity of serum acute-phase reactants (eg, erythrocyte-sedimentation rate [ESR] and CRP), and the inability of a negative temporal artery biopsy to exclude GCA definitively. Delayed or incomplete choroidal perfusion on fluorescein angiography (FA) 
has been reported as a highly suggestive indicator of active or impending AAION from GCA. ${ }^{5,8-11}$ The specificity of choroidal filling delay for the diagnosis of GCA in patients presenting with transient or permanent vision loss, however, remains unclear.

We report our experience of delayed choroidal filling on FA in a series of seven patients referred to an academic neuro-ophthalmology practice due to concern for GCA. Despite the FA findings, our examination, diagnostic testing, and long-term follow-up excluded the diagnosis of GCA in all cases, suggesting that choroidal perfusion abnormalities may occur in the absence of GCA.

\section{Patients and methods}

The charts of two academic neuro-ophthalmology practices were reviewed from January 2008 to January 2014. The Institutional Review Board of Washington University School of Medicine approved this study and formal patient consent was not sought considering it was a low-risk retrospective chart review using de-identified data. For inclusion in this study, patients demonstrated delayed, patchy choroidal filling on FA and underwent evaluation for GCA but were ultimately found to have an alternative diagnosis. History, clinical exam findings, acute-phase reactant results, neuroimaging, duration of follow-up, and temporal artery biopsies were examined. FA was performed on all patients within 10 days of symptom onset.

Retrospective independent review of the FA images by two authors was performed to determine the time to first choroidal filling and complete choroidal filling. Filling times were recorded as the average of the time at which the finding was first observed on FA with the time of the preceding frame. Any disparity between reviewers on these times was discussed until a consensus had been reached. The choroidal perfusion time, defined as the time from the first appearance of fluorescein in the choroid to complete filling of the choroid, was calculated. A summary of all seven cases is shown in Table 1, and we report two representative cases in more detail.

\section{Case report I}

A 55-year-old otherwise-healthy Caucasian man presented with 2 weeks of recurrent transient monocular vision-loss episodes in the left eye. He reported 16-18 episodes of generalized visual field constriction, with occasional photopsias progressing to near-complete visual loss, followed by a return to normal over 2 minutes. The patient denied headache, jaw claudication, scalp tenderness, fever, arthralgia, myalgia, and weight loss. Magnetic resonance imaging (MRI) of the brain and orbits with and without gadolinium, MR angiography of the head and neck, transthoracic echocardiography, electrocardiography, and carotid duplex studies were unremarkable. ESR was moderately elevated (60 mm/h).

Visual acuity was 20/20 in both eyes. Pupils were isocoric, briskly responsive to light, and did not demonstrate an afferent pupillary defect. Ophthalmoscopy demonstrated a cup:disk ratio of 0.75 in both eyes without edema or pallor and an otherwise normal fundus bilaterally. FA with transit images in the left eye showed a delay to initial filling (30.89 seconds), followed by prolonged patchy filling until 54.7 seconds (Figure 1). Calculated choroidal perfusion time was 23.81 seconds.

Oral prednisone ( $1 \mathrm{mg} / \mathrm{kg}$ daily) was started, and bilateral temporal artery biopsies were obtained within 1 week. Both biopsies were negative, and the patient was tapered off of oral prednisone quickly. On exam 6 weeks later, there was no change in visual acuity or the structural eye exam. A repeat FA showed normal, complete choroidal filling at 24 seconds in the left eye. The patient was diagnosed with idiopathic choroidal vasculopathy and remains asymptomatic, with no further episodes of vision loss on 4-year follow-up.

\section{Case report 2}

A 62-year-old Caucasian man with a history of non-insulindependent diabetes, hypertension, and coronary artery disease presented with acute vision loss in the left eye upon awakening. He reported an associated left frontal headache without jaw claudication, temple tenderness, or polymyalgia rheumatica symptoms.

Table I Summary of cases

\begin{tabular}{lllllllllll}
\hline Case & Sex & Age & ESR & CRP & TAB & Final diagnosis & $\begin{array}{l}\text { Follow-up } \\
\text { (months) }\end{array}$ & $\begin{array}{l}\text { First choroid } \\
\text { filling (seconds) }\end{array}$ & $\begin{array}{l}\text { Complete choroid } \\
\text { filling (seconds) }\end{array}$ & $\begin{array}{l}\text { Choroidal perfusion } \\
\text { time (seconds) }\end{array}$ \\
\hline I & M & 55 & 60 & NA & Neg & Choroidal vasculopathy & 57.5 & 30.89 & 54.7 & 2.8 \\
2 & M & 62 & 2 & $<3$ & Neg & NAION & 12 & 17.05 & 72.5 & 55.45 \\
3 & M & 61 & 16 & 24.8 & NA & NAION & 6.97 & 13 & 21.95 & 8.95 \\
4 & M & 88 & 72 & 27.5 & Neg & NAION, perioperative & 7.8 & 11.85 & 30.2 & 18.35 \\
5 & M & 50 & 7 & 1.7 & NA & NAION, perioperative & 9.97 & 10.55 & 18.4 & 7.85 \\
6 & F & 72 & 9 & 1.8 & Neg & NAION, sequential & 12.1 & 9.95 & 1.3 & 1.3 \\
7 & F & 75 & 12 & 1.4 & Neg & Migraine with visual aura & 11.97 & 18.15 & 28.45 & 1.3 \\
Average & 66.14 & & & & 16.90 & 15.92 & 35.35 & 19.43 \\
\hline
\end{tabular}

Abbreviations: ESR, erythrocyte-sedimentation rate; NA, not applicable; TAB, temporal artery biopsy; CRP, c-reactive protein; NAION, nonarteritic anterior ischemic optic neuropathy. 

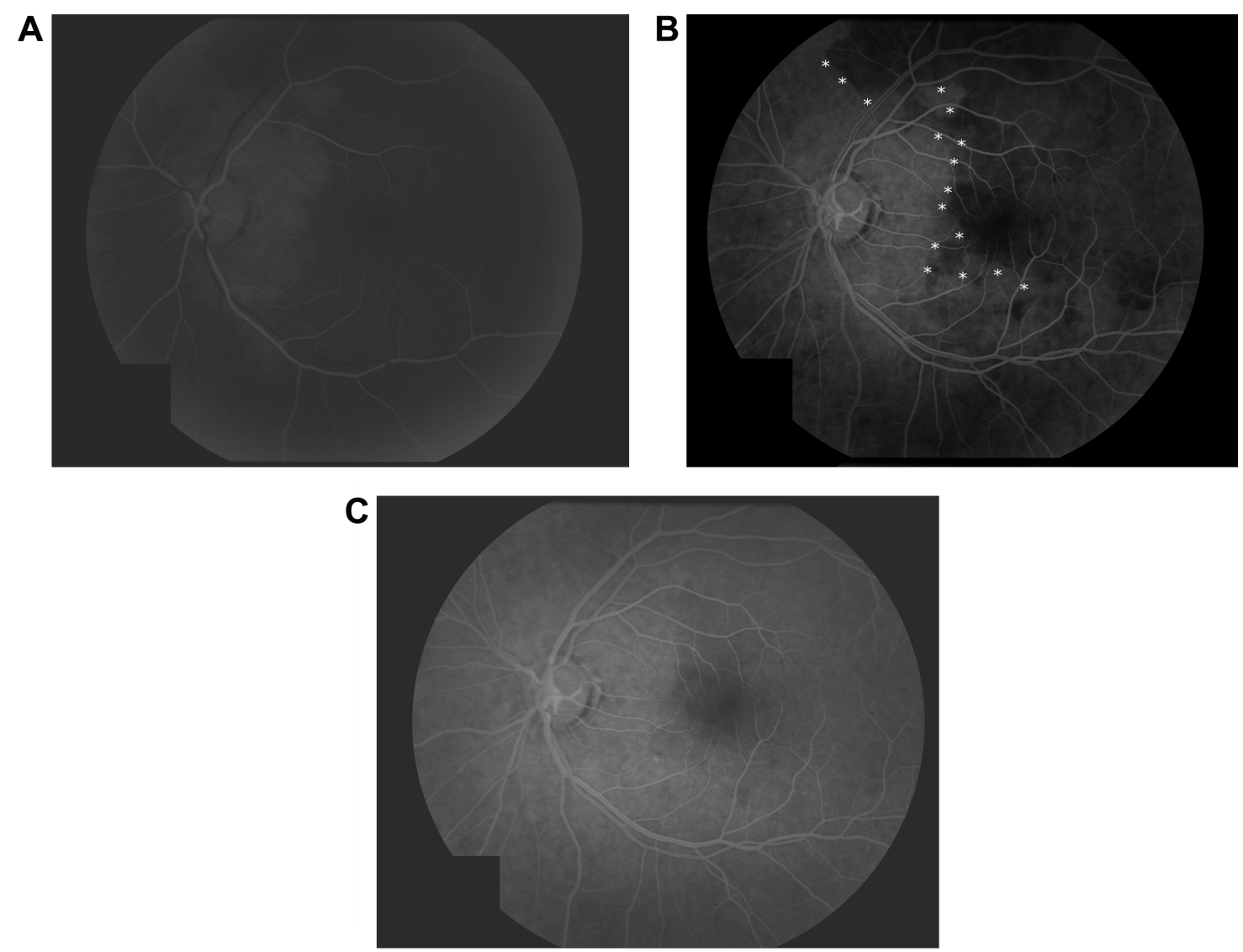

Figure I Case I transit fluorescein angiography images, left eye.

Notes: Patchy choroidal perfusion seen upon initial choroidal filling (A) at 30.89 seconds and persisting at 43.5 seconds (B). White asterisks highlight the demarcation line between normal choroidal filling nasally and patchy or absent choroidal filling temporally. Complete choroidal filling was achieved at 54.7 seconds (C). Calculated choroidal perfusion time was $23.8 \mathrm{I}$ seconds.

The referring provider exam showed that visual acuity was 20/20 in both eyes with a left afferent pupillary defect. Dilated fundus exam was remarkable for a normal right optic nerve with a cup:disk ratio of 0.25 and trace diffuse left optic disk swelling. Laboratory workup, including ESR $(2 \mathrm{~mm} / \mathrm{h})$ and CRP $(3 \mathrm{mg} / \mathrm{L})$, was normal. The next day, the patient returned for FA, which showed prominent choroidal filling delay of the nasal choroid (Figure 2). The calculated choroidal perfusion time was 55.45 seconds.

Over several days, the patient reported worsening of left frontal headaches and left-eye vision to the level of hand motion only. The left optic nerve showed moderate diffuse
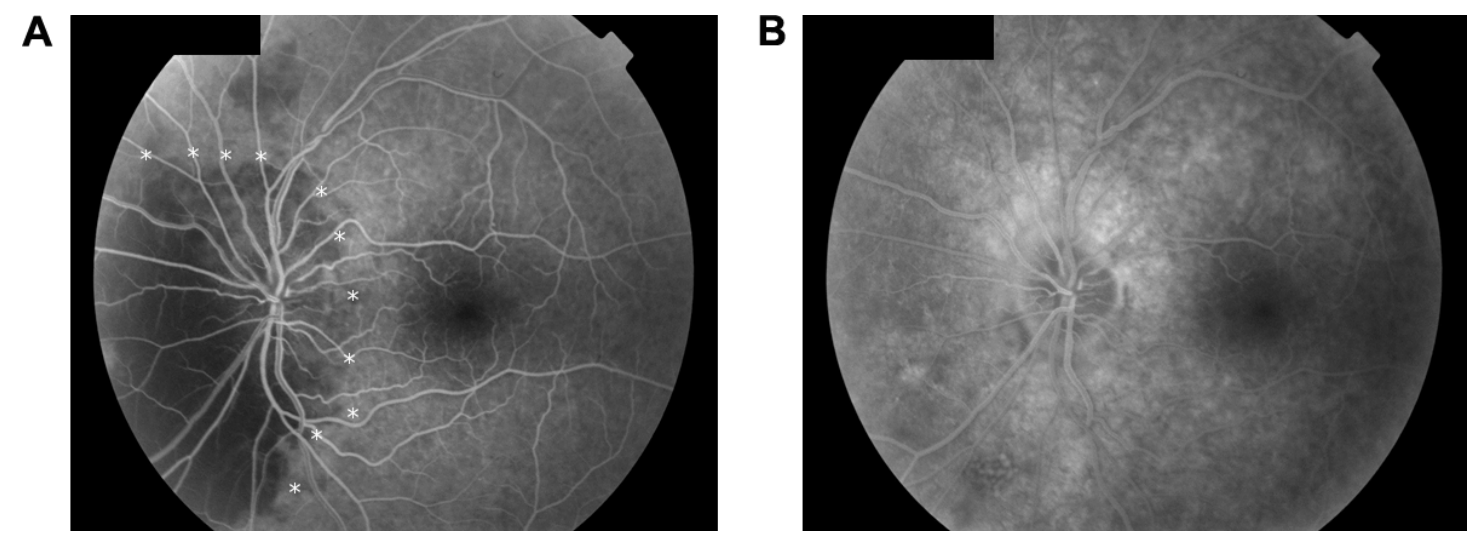

Figure 2 Case 2 transit fluorescein angiography images, left eye.

Notes: Delayed nasal choroidal filling at 30 seconds (A) and complete filling at 72.5 seconds (B). White asterisks highlight the demarcation line between normal choroidal filling temporally and patchy or absent choroidal filling nasally. Calculated choroidal perfusion time was 55.45 seconds. 
swelling with inferior peripapillary splinter hemorrhages. He was prescribed prednisone $80 \mathrm{mg}$ daily and underwent a negative left temporal artery biopsy. MRI of the brain and orbits with and without gadolinium was remarkable only for an incidental $1.2 \times 1 \mathrm{~cm}$ pituitary adenoma without compression of the anterior visual pathways. Two weeks later, visual acuity was stable in both eyes, headaches had resolved, and the left optic disk showed mild residual edema and early pallor. The patient was diagnosed with NAION. Twelve months later, the patient's vision remained stable, and he denied new vision changes or GCA symptoms.

\section{Results}

Upon chart review, seven patients (five men and two women, mean age 66.14 years) referred to an academic neuro-ophthalmology practice for possible GCA met studyinclusion criteria. Pertinent demographic information, diagnostic testing, final diagnosis, and FA filling times are displayed in Table 1. Three of seven patients had at least one elevated acute-phase reactant. Five of seven patients underwent unilateral or bilateral negative temporal artery biopsy. The mean time to first choroidal filling and complete choroidal filling was 15.92 (range 9.95-30.89) seconds and 35.35 (18.4-72.5) seconds, respectively. The mean choroidal perfusion time (interval from first to complete choroidal filling) was 19.43 (7.85-55.45) seconds. Five of seven patients were diagnosed with NAION, of which two occurred in the perioperative setting. One patient was diagnosed with migraine with visual aura and another with idiopathic choroidal vasculopathy. Mean follow-up time was 16.9 (7.8-57.5) months, with no new vision loss or symptom progression at final follow-up.

\section{Discussion}

AAION from GCA is approximately 20 times less common than NAION. ${ }^{12}$ Failure to establish a prompt diagnosis and initiate treatment with corticosteroids in unilateral AAION portends a poor visual prognosis for both eyes. Vision loss is bilateral simultaneous at presentation in an estimated $20 \%-62 \%$ of patients. Unilateral vision loss progresses to bilateral in up to $75 \%$ of untreated patients. ${ }^{13}$ Furthermore, systemic large-vessel cardiovascular morbidity in GCA due to aortic aneurysm, aortic dissection, cervical artery stenosis, and other sequelae has been reported in $27 \%$ of patients. ${ }^{7}$ Despite attempts to formulate an algorithmic approach to the diagnosis, the protean presentations and inherent shortcomings of testing for GCA can make it challenging for the clinician to recognize. ${ }^{14}$ Prior reports of prominent delayed choroidal filling in GCA suggest it is a useful diagnostic tool; however, the specificity for these findings remains unknown.

In normal individuals, intravenous fluorescein dye reaches the choroid via the ophthalmic artery, which gives rise to two to three main PCAs that subsequently subdivide into numerous short PCAs (SPCAs) supplying the optic nerve head and the choroid. Following injection of dye, choroidal filling in adolescents begins to occur at 8-12 seconds, while in older patients there may be a physiologic delay, with initial filling at $12-15$ seconds. ${ }^{15}$ While it may precede choroidal filling, the central retinal artery usually begins filling approximately $1-3$ seconds after dye is first seen in the choroid. ${ }^{15}$ Early choroidal filling in normal patients is often irregular and patchy, potentially due to the varying lengths of SPCAs supplying adjacent lobules. ${ }^{10}$ Terms defining choroidal perfusion in the literature may vary. The interval from the first appearance of dye in the retina or choroid to complete filling of all choroidal lobules has been termed by at least one author as the "choroidal filling time", while the interval between initial choroidal filling (regardless of retinal filling) and complete choroidal filling has been termed the "choroidal perfusion time". ${ }^{10,16}$ Considering retinal vascular filling usually follows choroidal filling, "choroidal filling time" and "choroidal perfusion time" are effectively the same in most patients. Normal physiologic choroidal filling time among controls (mean age 60 years) is $5.8 \pm 1.9$ seconds and choroidal perfusion time (mean age 64.6 years) $5.03 \pm 2.81$ seconds. ${ }^{10,16}$

In GCA-related AAION, localized thrombosis of the PCAs or more distal SPCAs results in choroidal perfusion delay or infarction of their respective vascular distributions. A watershed zone exists between the end-artery vascular territory of the medial and lateral PCAs, resulting in highly variable patterns of choroidal nonperfusion in GCA. When hypoperfusion involves the choroidal branches supplying the optic nerve head and meets a critical cytotoxic threshold, AAION occurs. Choroidal filling delay is a well-established indicator of GCA in the literature. ${ }^{5,11,17}$ Nevertheless, relatively few studies have defined what degree of choroidal perfusion delay strongly suggests GCA over alternative diagnoses.

Mack et al examined the FA of eleven patients with AAION due to biopsy-confirmed GCA and found the average choroidal filling time in this group was 69 (16-120) seconds versus $5.5(0-13.4)$ seconds in the NAION group $(P<0.001) .{ }^{10}$ In this study, a choroidal filling time of greater than 15-20 seconds appeared a highly suggestive indicator of the diagnosis of AAION, as opposed to NAION. ${ }^{10}$ Similarly to Mack et al, Arnold and Hepler found no statistical difference between choroidal perfusion time in 41 NAION 
patients (7.1 $\pm 7.97[0-48.1]$ seconds) compared to 43 agematched control patients (5.03 $\pm 2.81[0-12.6]$ seconds). ${ }^{16}$ Siatkowski et al compared FA characteristics of 16 patients with AAION due to biopsy-confirmed GCA to 16 patients with NAION. They found the mean choroidal filling time delay in AAION (29.7 16.9 [14-86] seconds) to be markedly longer and statistically significant compared to NAION $(12.9 \pm 3.1$ [7-18.8] seconds). ${ }^{9}$ Finally, Valmaggia et al prospectively compared FA and indocyanine green angiography in the diagnosis of AAION ( $n=5)$ compared to NAION $(n=17){ }^{8}$ The time from dye injection to complete choroidal filling was found to be markedly longer in AAION (39.8 \pm 22.2 [10.6-70] seconds) than NAION (18.5 \pm 5 [10.2-25] seconds). ${ }^{8}$

In our study, seven patients referred due to concern for GCA demonstrated patchy choroidal filling on FA, despite having alternative diagnoses (Table 1). The mean choroidal perfusion time in our patients (19.43 [7.85-55.45] seconds) was markedly delayed compared to published normals (5.03-5.08 seconds). ${ }^{10,16}$ Among our five patients with NAION, the mean choroidal perfusion time (20.38 [7.85-55.45] seconds) was prolonged compared to previously published choroidal filling times among NAION patients (7.1 \pm 7.97 [0-48.1] seconds). ${ }^{16}$ A review of prior studies, including Siatkowski et al and Valmaggia et al, shows that despite a statistically significant difference in the mean choroidal filling times between AAION in GCA and NAION, there was considerable overlap in the range of choroidal filling times exhibited in the two groups. ${ }^{8,9}$ This overlap exemplifies that while FA may be a helpful tool, it remains imperfect in its ability to distinguish GCA-related AAION from NAION. Indeed, in our five cases of NAION, all but case 2 had choroidal perfusion times that would have fallen within the range of NAION perfusion times reported by Siatkowski et al and Valmaggia et al. ${ }^{8,9}$ Most noteworthy from our series, patients 1 and 2 had markedly prolonged choroidal perfusion times that (based upon prior literature) would have been strongly indicative of GCA., ${ }^{9}$ These two cases demonstrate that markedly delayed choroidal filling can occur in the absence of GCA among patients being evaluated for the disease. Our study was limited by its small sample size, wide variability in presenting clinical features among patients, and the lack of an established gold standard in medicine for ruling out GCA.

Delayed choroidal perfusion is not specific for GCA and can be seen in multiple other diagnoses. Ipsilateral high-grade stenosis of the internal carotid artery can manifest in markedly delayed perfusion through the PCAs, with resultant choroidal perfusion delay or infarction. Most cases of carotid stenosis will have other sequelae of ocular ischemia syndrome, including mid-peripheral retinal hemorrhages, dilated retinal veins, retinal neovascularization, or anterior-segment ischemia. ${ }^{18}$ Severe hypertension, regardless of etiology (eg, essential, toxemia of pregnancy, thrombocytopenic purpura, diffuse intravascular coagulation, renal failure), can result in focal ischemic infarcts of the choroid, referred to by some as Elschnig spots. Patients with high blood pressure-related choroidal perfusion abnormalities typically demonstrate additional hypertensive retinal findings. ${ }^{19-21}$ In hypertensive serous retinal detachment, choroidal hypoperfusion on FA often underlies the detachment, suggesting that choroidal ischemia and resultant retinal pigment epithelium edema may cause the detachment. ${ }^{19}$ While a rare cause of choroidal ischemia compared to GCA, granulomatosis with polyangiitis (formerly termed Wegener's granulomatosis) has been reported to cause choroidal ischemia. ${ }^{22,23}$ Biopsy-supported varicella zoster vasculopathy has also been reported to affect PCAs, with FA findings of choroidal hypoperfusion. ${ }^{24}$ Patients with hemoglobinopathies, such as sickle-cell disease and sickle-cell thalassemia variants, can experience choroidal infarction following photocoagulation treatment. ${ }^{25}$

\section{Conclusion}

Similarly to other imperfect tests for GCA, delayed choroidal filling on FA is suggestive but not completely specific for the disease. In the correct clinical context, an interval greater than 15-20 seconds from initial to complete choroidal filling is supportive of a diagnosis of GCA. Our series highlights that choroidal filling delay in older patients presenting with acute vision loss in the absence of GCA is not rare; furthermore, even massively delayed choroidal filling is not diagnostic of GCA. When evaluating a patient for acute vision loss, the astute clinician must remain cognizant of the limitations of FA in the diagnosis of GCA.

\section{Acknowledgments}

The research in this paper was presented in preliminary form at the North American Neuro-Ophthalmology Society (NANOS) Annual General Meeting, March 1-6, 2014, in Rio Grande, Puerto Rico, as a poster presentation. The poster abstract was published as "Poster 231" in the meeting syllabus.

\section{Disclosure}

The authors report no conflicts of interest in this work.

\section{References}

1. Lawrence RC, Helmick CG, Arnett FC, et al. Estimates of the prevalence of arthritis and selected musculoskeletal disorders in the United States. Arthritis Rheum. 1998;41(5):778-799. 
2. Machado EB, Michet CJ, Ballard DJ, et al. Trends in incidence and clinical presentation of temporal arteritis in Olmsted County, Minnesota, 1950-1985. Arthritis Rheum. 1988;31(6):745-749.

3. Liu GT, Glaser JS, Schatz NJ, Smith JL. Visual morbidity in giant-cell arteritis: clinical characteristics and prognosis for vision. Ophthalmology. 1994;101(11):1779-1785.

4. Foroozan R, Deramo VA, Buono LM, et al. Recovery of visual function in patients with biopsy-proven giant cell arteritis. Ophthalmology. 2003;110(3):539-542.

5. Chew SS, Kerr NM, Danesh-Meyer HV. Giant cell arteritis. J Clin Neurosci. 2009;16(10):1263-1268.

6. Lie JT. Aortic and extracranial large vessel giant cell arteritis: a review of 72 cases with histopathologic documentation. Semin Arthritis Rheum. 1995;24(6):422-431.

7. Nuenninghoff DM, Hunder GG, Christianson TJ, McClelland RL, Matteson EL. Incidence and predictors of large-artery complication (aortic aneurysm, aortic dissection, and/or large-artery stenosis) in patients with giant cell arteritis: a population-based study over 50 years. Arthritis Rheum. 2003;48(12):3522-3531.

8. Valmaggia C, Speiser P, Bischoff P, Niederberger H. Indocyanine green versus fluorescein angiography in the differential diagnosis of arteritic and nonarteritic anterior ischemic optic neuropathy. Retina. 1999;19(2):131-134.

9. Siatkowski RM, Gass JD, Glaser JS, Smith JL, Schatz NJ, Schiffman J. Fluorescein angiography in the diagnosis of giant cell arteritis. Am J Ophthalmol. 1993;115(1):57-63.

10. Mack HG, O’Day J, Currie JN. Delayed choroidal perfusion in giant cell arteritis. J Clin Neuroophthalmol. 1991;11(4):221-227.

11. Hayreh SS. Anterior ischemic optic neuropathy: differentiation of arteritic from non-arteritic type and its management. Eye (Lond). 1990;4(Pt 1): $25-41$.

12. Guyer DR, Miller NR, Auer CL, Fine SL. The risk of cerebrovascular and cardiovascular disease in patients with anterior ischemic optic neuropathy. Arch Ophthalmol. 1985;103(8):1136-1142.
13. Jonasson F, Cullen JF, Elton RA. Temporal arteritis: a 14-year epidemiological, clinical and prognostic study. Scott Med J. 1979;24(2):111-117.

14. El-Dairi MA, Chang L, Proia AD, Cummings TJ, Stinnett SS, Bhatti MT. Diagnostic algorithm for patients with suspected giant cell arteritis. J Neuroophthalmol. 2015;35(3):246-253.

15. Ryan SJ. Retina. 5th ed. London: Saunders/Elsevier; 2013.

16. Arnold AC, Hepler RS. Fluorescein angiography in acute nonarteritic anterior ischemic optic neuropathy. Am J Ophthalmol. 1994;117(2): 222-230.

17. Slavin ML, Barondes MJ. Visual loss caused by choroidal ischemia preceding anterior ischemic optic neuropathy in giant cell arteritis. Am J Ophthalmol. 1994;117(1):81-86.

18. Ong TJ, Paine M, O'Day J. Retinal manifestations of ophthalmic artery hypoperfusion. Clin Experiment Ophthalmol. 2002;30(4):284-291.

19. Fastenberg DM, Fetkenhour CL, Choromokos E, Shoch DE. Choroidal vascular changes in toxemia of pregnancy. Am JOphthalmol. 1980;89(3): 362-368.

20. Hayreh SS, Servais GE, Virdi PS. Fundus lesions in malignant hypertension: VI - hypertensive choroidopathy. Ophthalmology. 1986;93(11): 1383-1400.

21. Klien BA. Ischemic infarcts of the choroid (Elschnig spots): a cause of retinal separation in hypertensive disease with renal insufficiency a clinical and histopathologic study. Am J Ophthalmol. 1968;66(6): 1069-1074.

22. Iida T, Spaide RF, Kantor J. Retinal and choroidal arterial occlusion in Wegener's granulomatosis. Am J Ophthalmol. 2002;133(1):151-152.

23. Kinyoun JL, Kalina RE, Klein ML. Choroidal involvement in systemic necrotizing vasculitis. Arch Ophthalmol. 1987;105(7):939-942.

24. Salazar R, Russman AN, Nagel MA, et al. Varicella zoster virus ischemic optic neuropathy and subclinical temporal artery involvement. Arch Neurol. 2011;68(4):517-520.

25. Goldbaum MH, Galinos SO, Apple D, et al. Acute choroidal ischemia as a complication of photocoagulation. Arch Ophthalmol. 1976;94(6): 1025-1035.
Clinical Ophthalmology

\section{Publish your work in this journal}

Clinical Ophthalmology is an international, peer-reviewed journal covering all subspecialties within ophthalmology. Key topics include: Optometry; Visual science; Pharmacology and drug therapy in eye diseases; Basic Sciences; Primary and Secondary eye care; Patient Safety and Quality of Care Improvements. This journal is indexed on Submit your manuscript here: http://www.dovepress.com/clinical-ophthalmology-journal

\section{Dovepress}

PubMed Central and CAS, and is the official journal of The Society of Clinical Ophthalmology (SCO). The manuscript management system is completely online and includes a very quick and fair peer-review system, which is all easy to use. Visit http://www.dovepress.com/ testimonials.php to read real quotes from published authors. 